\title{
Impact of renin-angiotensin-aldosterone system gene polymorphisms on left ventricular dysfunction in coronary artery disease patients
}

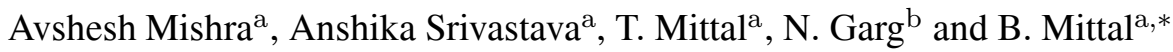 \\ ${ }^{a}$ Department of Genetics, Sanjay Gandhi Post Graduate Institute of Medical Sciences (SGPGIMS), Lucknow, India \\ ${ }^{\mathrm{b}}$ Department of Cardiology, Sanjay Gandhi Post Graduate Institute of Medical Sciences (SGPGIMS), Lucknow, \\ India
}

\begin{abstract}
Background: Left ventricular dysfunction (LVD), followed by fall in cardiac output is one of the major complications in some coronary artery disease (CAD) patients. The decreased cardiac output over time leads to activation of the renin-angiotensinaldosterone system which results in vasoconstriction by influencing salt-water homeostasis. Therefore, the purpose of the present study was to explore the association of single nucleotide polymorphisms (SNPs) in angiotensin I converting enzyme; $A C E$ (rs4340), angiotensin II type1 receptor; AT1 (rs5186) and aldosterone synthase; CYP11B2 (rs1799998) with LVD.

Methods and results: The present study was carried out in two cohorts. The primary cohort included 308 consecutive patients with angiographically confirmed CAD and 234 healthy controls. Among CAD, 94 with compromised left ventricle ejection fraction (LVEF $\leqslant 45$ ) were categorized as LVD. The ACE I/D, AT1 A1166C and CYP11B2 T-344C polymorphisms were determined by PCR. Our results showed that $A C E$ I/D was significantly associated with CAD but not with LVD. However, AT1 1166C variant was significantly associated with LVD $(\mathrm{LVEF} \leqslant 45)(\mathrm{p}$ value $=0.013 ; \mathrm{OR}=3.69)$, but $C Y P 11 B 2$ (rs 1799998) was not associated with either CAD or LVD. To validate our results, we performed a replication study in additional 200 cases with similar clinical characteristics and results again confirmed consistent findings ( $\mathrm{p}$ value $=0.020$; OR $=5.20$ ).

Conclusion: AT1 A1166C plays important role in conferring susceptibility of LVD.
\end{abstract}

Keywords: Coronary artery disease, LVD, LVEF, RAAS genetic variants

\section{Introduction}

Coronary artery disease (CAD) is a complex, multifactorial disease, influenced by pathophysiologic conditions as well as by genetic and environmental factors [1]. The major complication that some CAD patients face over time is the impairment in left ventricular function, followed by fall in cardiac output. Despite being progressively debilitating, this condition does not

* Corresponding author: Dr. Balraj Mittal (Professor), Department of Genetics, SGPGIMS, Lucknow-226014 (UP) India. Fax: +91 522 2494322; E-mail: balraj@sgpgi.ac.in/bml_pgi@yahoo.com. affect everybody equally [2] and genetic differences may provide an explanation for the fact that some people, irrespective of lifestyle and common classical cardiovascular risk factors, are more prone to develop Left Ventricle Dysfunction (LVD) than others.

Clinically, LVD is defined as a syndrome that develops in response to an ischemic insult, resulting in decline in the pumping capacity of the heart. This is furthermore characterized by the continuous interactions between the underlying myocardial dysfunction and subsequent compensatory neurohumoral mechanisms that are activated [3]. In view of its physiological role and established benefits of ACE inhibitors therapy, 
attention has been focused on the renin-angiotensinaldosterone system (RAAS) [4]. This system is initially able to compensate for the depressed myocardial function and preserve the cardiovascular homeostasis. However, their long-term activation has deleterious effects on cardiac structure and performance, leading to cardiac decompensation and progression of heart failure. In patients with cardiovascular disease, activity of the RAAS is often increased and contributes to a poor prognosis [5].

In RAAS cascade, angiotensinogen (AGT) is cleaved by renin to produce angiotensin I, which is further converted in the bioactive octapeptide angiotensin II (AngII) through the action of angiotensin I converting enzyme (ACE), a membrane-bound, zinc metalloendopeptidase involved in the metabolism of many small peptides. Many of the known deleterious effects of Ang II relevant to cardiovascular function and structure are mediated by angiotensin II type1 (AT1) receptor stimulation. These effects include vasoconstriction, sodium and water retention, aldosterone release, augmentation of sympathetic nervous system activity and vascular hypertrophy.

In addition, aldosterone synthase (CYP11B2), sensitive to the effects of ATII, catalyses the final step of aldosterone biosynthesis and release from zona glomerulosa in adrenal cortex. Because of the range of physiologic effects of $A C E$, $A T 1$ and $C Y P 11 B 2$, genetic variations that affect baseline RAAS activity are, therefore, candidates for increasing risk of, and adverse outcomes in heart disease and could probably affect a wide variety of clinical phenotypes. Therefore, we undertook the present study to assess the association of $A C E$ insertion/deletion (ACE I/D rs4340), AT1 (A1166C rs5186) and $C Y P 11 B 2$ (T-344C rs1799998) polymorphisms with $\mathrm{LV}$ dysfunction in CAD patients. To the best of our knowledge, this is the first report investigating the association between $A C E, A T 1$ and $C Y P 11 B 2$ gene polymorphism with left ventricular dysfunction in coronary artery disease patients.

\section{Materials and methods}

\subsection{Study population}

The present study was carried out in two cohorts, primary and replication cohorts. In the primary cohort, we studied 308 CAD patients recruited from July 2008 to January 2010. In the replication cohort, further 200 cases were enrolled. The diagnostic parameters used in the primary cohort, were also applied to the replication cohort. Both the primary and replication cohorts had significant coronary artery disease, (diagnosis, confirmed by coronary angiography and further all these subjects underwent coronary angioplasty) recruited from the Department of Cardiology of Sanjay Gandhi Postgraduate Institute of Medical Sciences (SGPGIMS), Lucknow, Uttar Pradesh, India. The detailed clinical history of CAD patients was based on hospital investigations including coronary angiography. Angiographically identified stenoses $>70 \%$ in the major coronary vessels at the time of the study were used to classify patients as having single-vessel, double-vessel, or triple-vessel disease. The control (non-CAD) population consisted of 234 subjects (187 males and $47 \mathrm{fe}-$ males) (mean age years $54.18 \pm 8.47$ ) with no clinical evidence of CAD or LV dysfunction (by echocardiography) and also without positive family history of CAD or myocardial infarction (MI). Furthermore, the inclusion criteria for controls were absence of prior history of high systolic blood pressure, abnormal lipid profile, hypertension and obesity. Both patients and controls were frequency-matched to age, gender and ethnicity. To test the possibility for population stratification, genomic control method was used as described by Devlin et al. [6]. After obtaining informed consent, all the individuals were personally interviewed for information on food habits, occupation and tobacco usage. The study was approved by local ethical review committees of the institute and the authors followed the norms of World's Association Declaration of Helsinki [7].

\subsection{Data collection}

The clinical data was obtained by reviewing the patient's medical records. Left ventricle ejection fraction (LVEF) was calculated quantitatively by echocardiography, just before angiography procedure, using the Simpson's method [8]. Echocardiography was repeated in $10 \%$ of patients and results were totally concordant. Hypertension was defined as systolic blood pressure $>140 \mathrm{mmHg}$ or a diastolic blood pressure $>90 \mathrm{mmHg}$ or patients using antihypertensive drugs. Smoking was classified as smokers (ex-smoker and current smokers) and non smokers. Similarly, diabetes mellitus was defined as patients with fasting plasma glucose $>6.9 \mathrm{mmol} / \mathrm{L}$ or patients using anti-diabetic medication. All laboratory parameters, as stated in the medical record, were determined in overnight-fasting patients. Total cholesterol, high-density lipoprotein (HDL) cholesterol and triglyceride levels were mea- 
Table 1

Clinical characteristics of CAD patients

\begin{tabular}{|c|c|c|c|}
\hline Clinical characteristics & Primary stage & Replication stage & Combined \\
\hline Total subjects & 308 & 200 & 508 \\
\hline *Age-yr & $56.26 \pm 9.78$ & $55.69 \pm 8.88$ & $56.04 \pm 9.42$ \\
\hline Male sex & $260(84.4 \%)$ & $179(89.5 \%)$ & $439(86.4 \%)$ \\
\hline \multicolumn{4}{|l|}{ Risk factors } \\
\hline Hypertension & $147(47.7 \%)$ & $85(42.5 \%)$ & $232(45.7 \%)$ \\
\hline Diabetes & $91(29.5 \%)$ & $59(29.5 \%)$ & $150(29.5 \%)$ \\
\hline Smoking & $78(25.3 \%)$ & $54(27 \%)$ & $132(26 \%)$ \\
\hline BMI & $24.39 \pm 3.36$ & $24.49 \pm 2.97$ & $24.45 \pm 3.13$ \\
\hline \multicolumn{4}{|l|}{${ }^{*}$ Lipid levels } \\
\hline A) High density lipoprotein (mg/dl) & $32.31 \pm 7.91$ & $31.88 \pm 5.86$ & $32.19 \pm 7.45$ \\
\hline B) Low density lipoprotein (mg/dl) & $100.13 \pm 24.33$ & $103.74 \pm 22.90$ & $100.97 \pm 24.40$ \\
\hline C) Triglycerides (mg/dl) & $155.64 \pm 69.19$ & $125.41 \pm 35.54$ & $149.11 \pm 62.25$ \\
\hline D) Total cholesterol (mg/dl) & $170.30 \pm 21.98$ & $141.75 \pm 39.61$ & $163.74 \pm 30.53$ \\
\hline \multicolumn{4}{|l|}{ Clinical syndrome } \\
\hline Stable angina & $91(29.5 \%)$ & $63(31.5 \%)$ & $156(30.7 \%)$ \\
\hline Unstable angina/non st elevation myocardial infarction (NSTEMI) & $69(22.3 \%)$ & $40(20.5 \%)$ & $109(21.5 \%)$ \\
\hline St elevation myocardial infarction (STEMI) & $148(48.2 \%)$ & $96(48.0 \%)$ & $242(47.7 \%)$ \\
\hline Anterior wall myocardial infarction (AWMI) & $84(27.3 \%)$ & $47(23.5 \%)$ & $130(25.6 \%)$ \\
\hline Inferior wall myocardial infarction (IWMI) & $63(20.5 \%)$ & $49(24.5 \%)$ & $110(21.7 \%)$ \\
\hline Lateral wall myocardial infarction (LWMI) & $2(0.4 \%)$ & $0(0.0 \%)$ & $2(0.4 \%)$ \\
\hline \multicolumn{4}{|l|}{ Angiographic profile } \\
\hline Single vessel disease (SVD) & $200(64.9 \%)$ & $141(70.0 \%)$ & $341(67.2 \%)$ \\
\hline Double vessel disease (DVD) & $85(27.6 \%)$ & $27(13.5 \%)$ & $112(22.0 \%)$ \\
\hline Triple vessel disease (TVD) & $23(7.5 \%)$ & $32(16.5 \%)$ & $55(10.8 \%)$ \\
\hline \multicolumn{4}{|l|}{ Left ventricular function } \\
\hline${ }^{*}$ mean left ventricle ejection fraction (LVEF) & $50.70 \pm 11.65$ & $48.56 \pm 10.2$ & $50.0 \pm 11.18$ \\
\hline$>45$ & $214(69.5 \%)$ & $127(63.5 \%)$ & $341(67.1 \%)$ \\
\hline$\leqslant 45$ & $94(30.5 \%)$ & $73(36.5 \%)$ & $167(32.9 \%)$ \\
\hline
\end{tabular}

*Values are mean $\pm \mathrm{SD}$.

sured by standard enzymatic methods. LDL cholesterol concentrations were calculated using the Friedewald's formula [9].

\subsection{Genotyping}

Genomic DNA was isolated from peripheral blood leukocytes according to a standard salting out method [10]. The polymorphisms were genotyped using the PCR or PCR-restriction fragment length polymorphism method. As a negative control, PCR mix without DNA sample was used to ensure contamination free PCR product. Genotyping of ACE I/D was based on the PCR amplification of a fragment encompassing the 287 bp insertion polymorphism in intron 16 as described by Joshi et al. [11]. Furthermore, the genotyping of AT1 A1166C was determined by allele specific primers described by Shu Ye et al. [12] whereas CYP11B2 T344C polymorphisms were determined by using the polymerase chain reaction (PCR)-restriction fragment length polymorphism method as described by Kupari et al. [13]. The digested PCR fragments were separated on $2 \%$ agarose gel, stained with ethidium bromide and observed with ultraviolet imaging system (Vilber
Lourmat, Marne-la-Valle'e, France). Genotyping was performed without knowledge of the case or control status. Ten percent of samples for each genotype were sequenced which showed 100\% concordance.

\subsection{Statistical analysis}

The sample size was calculated using QUANTO 1.1, using minor allele frequency data from HapMap (http://www.hapmap.org/). The sample size of both primary (308) and replication (200) cohorts and 234 controls were adequate to give us power of $80 \%$ (probability of not making a type II error).

Descriptive statistics were presented as mean and standard deviation (SD) for continuous measures while absolute value and percentages were used for categorical measures. The chi-square goodness of fit test was used for any deviation from Hardy Weinberg Equilibrium in controls. Differences in genotype and allele frequencies between study groups were estimated by chi-square test. The ORs were adjusted for confounding factors such as age and gender. In addition, the association between RAAS gene polymorphisms and significant risk factors of CAD were analyzed using 
Table 2

Distributions for RAAS gene Polymorphism in CAD patients and Healthy controls

\begin{tabular}{|c|c|c|c|c|c|}
\hline Genotypes/Alleles & $\mathrm{HC}(234) \mathrm{N}(\%)$ & $\mathrm{CAD}^{\mathrm{a}}(308) \mathrm{N}(\%)$ & $\mathrm{CAD}^{\mathrm{b}}(200) \mathrm{N}(\%)$ & $\mathrm{P}$ value $^{\mathrm{a}}$ OR $(95 \% \mathrm{CI})$ & P-value ${ }^{\mathrm{b}}$ OR $(95 \% \mathrm{CI})$ \\
\hline \multicolumn{6}{|l|}{ ACE I/D } \\
\hline II & $103(44)$ & $99(32.1)$ & $79(39.5)$ & 1 (reference) & 1 (reference) \\
\hline ID & $112(47.9)$ & $141(45.8)$ & $80(40.0)$ & $0.1541 .3(0.9-1.8)$ & $0.7340 .93(0.62-1.40)$ \\
\hline DD & $19(8.1)$ & $68(22.1)$ & $41(20.5)$ & $<0.0013 .72(2.08-6.64)$ & $0.0012 .81(1.52-5.22)$ \\
\hline I & $318(67.9)$ & $339(55.0)$ & $238(59.5)$ & 1 (reference) & 1 (reference) \\
\hline $\mathrm{D}$ & $150(32.1)$ & $277(45.0)$ & $162(40.5)$ & $<0.0011 .78(1.38-2.29)$ & $0.0411 .37(0.99-1.88)$ \\
\hline \multicolumn{6}{|l|}{ AT1 A1166C } \\
\hline AA & $168(71.8)$ & $232(75.3)$ & $155(77.5)$ & 1 (reference) & 1 (reference) \\
\hline $\mathrm{AC}$ & $56(23.9)$ & $60(19.5)$ & $35(17.5)$ & $0.2300 .78(0.51-1.17)$ & $0.1080 .68(0.42-1.09)$ \\
\hline $\mathrm{CC}$ & $10(4.3)$ & $16(5.2)$ & $10(5.0)$ & $0.7231 .16(0.51-2.62)$ & $0.8611 .08(0.44-2.67)$ \\
\hline A & $392(83.8)$ & $524(85.1)$ & $345(86.2)$ & 1 (reference) & 1 (reference) \\
\hline $\mathrm{C}$ & $76(16.2)$ & $92(14.9)$ & $55(17.8)$ & $0.4460 .88(0.63-1.23)$ & $0.2310 .77(0.50-1.19)$ \\
\hline \multicolumn{6}{|l|}{ CYP11B2 T-344C } \\
\hline TT & $77(32.9)$ & $113(36.7)$ & $77(38.5)$ & 1 (reference) & 1 (reference) \\
\hline $\mathrm{TC}$ & $124(53)$ & $151(49.0)$ & $101(50.5)$ & $0.3290 .83(0.57-1.20)$ & $0.3280 .82(0.54-1.23)$ \\
\hline $\mathrm{CC}$ & $33(14.1)$ & $44(14.3)$ & $22(11.0)$ & $0.7260 .91(0.53-1.55)$ & $0.2040 .67(0.36-1.25)$ \\
\hline $\mathrm{T}$ & $278(59.4)$ & $377(61.2)$ & $255(63.8)$ & 1 (reference) & 1 (reference) \\
\hline $\mathrm{C}$ & $190(40.6)$ & $239(38.8)$ & $145(36.2)$ & $0.7350 .88(0.43-1.81)$ & $0.6120 .92(0.68-1.26)$ \\
\hline
\end{tabular}

CAD-Coronary Artery Disease, HC-healthy control, OR-odds ratio, CI-confidence interval; D,C,C are variant allele; I,A,T are wild-type allele for ACE,AT1 and CYP111B2 respectively; ${ }^{\mathrm{a}} \mathrm{CAD}$ patients in primary cohort; ${ }^{\mathrm{b}} \mathrm{CAD}$ patients in replication cohort;

$a=$ represents the $\mathrm{p}$ values for the comparison in CAD patients (primary cohort) and $\mathrm{HC}$;

$b=$ represents the $\mathrm{p}$ values for the comparison in CAD patients (replication cohort) and HC.

Significant values are shown in bold.

binary logistic regression. Overall we performed meta analysis by Stouffers method to combine the results of two cohorts (primary and replication) to calculate the overall $\mathrm{z}$ and $\mathrm{p}$ values. A two-tailed $\mathrm{p}$-value of less than 0.05 was considered as statistical significant result. All statistical analyses were performed using SPSS software version 16.0 (SPSS, Chicago, IL, USA).

\section{Results}

\subsection{Patient characteristics}

Clinical characteristics of CAD patients-Primary, replication and combined cohorts of study are shown in Table 1. There was no significant difference in the mean age of CAD patients and controls. The male/female ratio was comparable in both $\mathrm{CAD}$ cases as well as in controls. Evaluation of the defined risk factors in the cohort showed that $45.7 \%$ patients were hypertensive and $29.5 \%$ patients were diabetic. Moreover, $26.0 \%$ patients were associated with tobacco usage. Patients with stable angina were $30.7 \%$ and unstable angina/ Non ST Segment Elevation Myocardial Infarction (NSTEMI) formed $21.5 \%$ of the clinical syndrome. ST Segment Elevation Myocardial Infarction (STEMI) patients with anterior wall myocardial infarction (AWMI) and inferior wall myocardial infarction (IWMI) were $25.6 \%$ and $21.7 \%$ respectively. Only two patients were found to be affected with lateral wall myocardial infarction (LWMI) $(0.4 \%)$. The angiographic profile categorized patients with single vessel disease (SVD), double vessel disease (DVD), and triple vessel disease (TVD) as $67.2 \%, 22.0 \%$ and $10.8 \%$ respectively. The mean ejection fraction was $50.0 \pm 11.18$. The kurtosis and skewness for left ventricular ejection fraction (LVEF) were 0.12 and -0.98 . Thus, the data appeared to be normally distributed. Although in clinical practice, LVEF of $<50 \%$ is considered as LVD but we selected $\leqslant 45 \%$ cut off as significant LVD. Of the total 508 CAD patients, $67.1 \%$ showed preserved $(>45 \%)$ ejection fraction while $32.9 \%$ had compromised ejection fractions $(\leqslant 45 \%)$. All the values in primary cohort matched to replication cohort and there was no significant difference in the values of three cohorts of the study.

\subsection{Allelic distribution of studied polymorphisms in controls}

The distribution of $A C E$ I/D, ATI A1166C and CYP11B2 T-344C genotypes is shown in Table 2 . The observed genotype frequencies of all the studied polymorphisms in healthy controls were in accordance with Hardy-Weinberg equilibrium $(p>0.05)$. 
Table $3 \mathrm{a}$

Distributions of RAAS gene polymorphisms in primary cohort of CAD patients with preserved (LVEF > 45) and compromised (LVEF $\leqslant 45)$ left ventricular ejection fraction

\begin{tabular}{|c|c|c|c|c|}
\hline LVEF categorical & Genotypes & CAD N (\%) 308 & P-value & OR $(95 \% \mathrm{CI})$ \\
\hline \multicolumn{5}{|l|}{$A C E \mathbf{I} / \mathbf{D}$} \\
\hline \multirow[t]{3}{*}{$>45$} & II & $68(31.8)$ & \multicolumn{2}{|r|}{ Reference } \\
\hline & ID & $98(45.8)$ & & \\
\hline & DD & $48(22.4)$ & & \\
\hline \multirow[t]{3}{*}{$\leqslant 45$} & II & $31(33.0)$ & - & - \\
\hline & ID & $43(45.7)$ & 0.89 & $0.96(0.55-1.68)$ \\
\hline & DD & $20(21.3)$ & 0.79 & $0.91(0.47-1.80)$ \\
\hline \multicolumn{5}{|l|}{$A T 1$ A1166C } \\
\hline \multirow[t]{3}{*}{$>45$} & AA & $172(80.4)$ & \multirow{3}{*}{\multicolumn{2}{|c|}{ Reference }} \\
\hline & $\mathrm{AC}$ & $35(16.3)$ & & \\
\hline & $\mathrm{CC}$ & $7(3.3)$ & & \\
\hline \multirow[t]{3}{*}{$\leqslant 45$} & AA & $60(63.8)$ & - & - \\
\hline & $\mathrm{AC}$ & $25(26.6)$ & 0.018 & $2.05(1.13-3.70)$ \\
\hline & $\mathrm{CC}$ & $9(9.6)$ & 0.013 & $3.69(1.32-10.33)$ \\
\hline \multicolumn{5}{|l|}{ CYP11B2 T-344C } \\
\hline \multirow[t]{3}{*}{$>45$} & TT & $80(37.4)$ & \multirow{3}{*}{\multicolumn{2}{|c|}{ Reference }} \\
\hline & $\mathrm{TC}$ & $102(47.6)$ & & \\
\hline & $\mathrm{CC}$ & $32(15.0)$ & & \\
\hline \multirow[t]{3}{*}{$\leqslant 45$} & TT & $33(35.1)$ & - & - \\
\hline & $\mathrm{TC}$ & $49(52.1)$ & 0.573 & $1.16(0.69-1.98)$ \\
\hline & $\mathrm{CC}$ & $12(12.8)$ & 0.810 & $0.91(0.42-1.97)$ \\
\hline
\end{tabular}

Table $3 b$

Significant values are shown in bold.

Distributions of RAAS gene polymorphisms in replication cohort of CAD patients with preserved $(\mathrm{LVEF}>45)$ and compromised $(\mathrm{LVEF} \leqslant 45)$ left ventricular ejection fraction

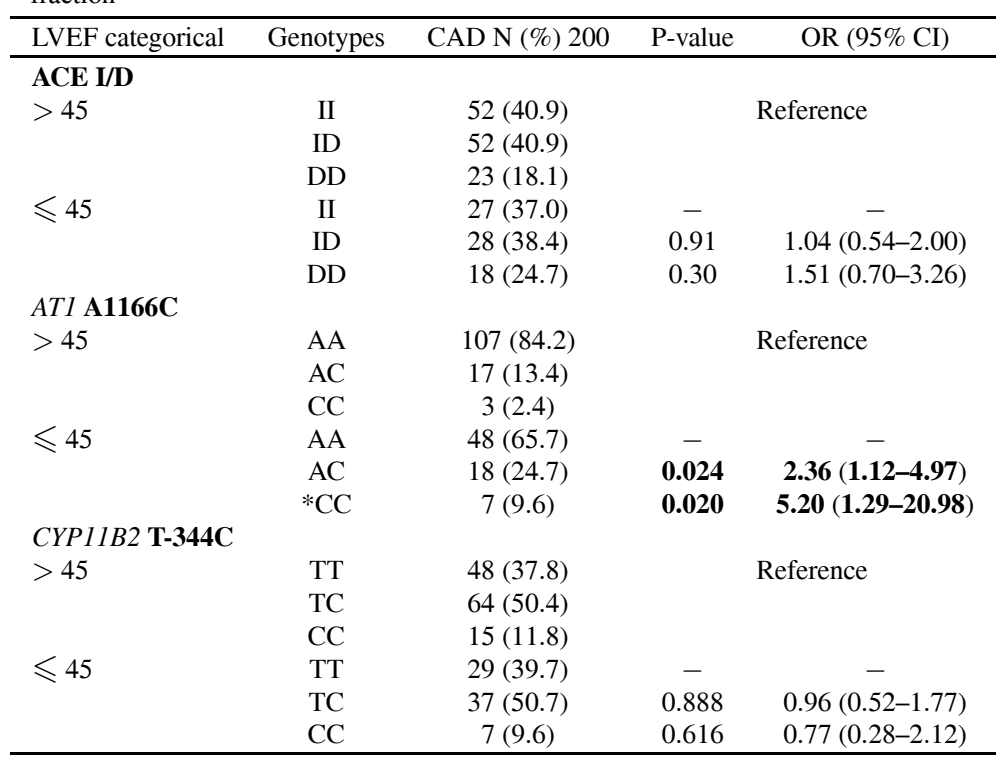

* Overall $\mathrm{z}$ value for primary and replicative cohort $=3.02 ; \mathrm{P}$ value $\leqslant 0.001$.

Significant values are shown in bold.

3.3. Frequency distribution of RAAS gene polymorphism in CAD patients and controls

Table 2 shows the risk of CAD in relation to each of the SNPs of RAAS genes. On comparing the genotype frequency distribution in CAD patients (both cohorts) with that of controls, the homozygous variant genotypes (DD) of ACE I/D and D allele showed statistically significant increased risk for developing $\operatorname{CAD}[(p \leqslant$ 
Table 4

Distributions for AT1 A1166C gene Polymorphism in STEMI subjects with preserved $(\mathrm{LVEF}>45)$ and compromised $(\mathrm{LVEF} \leqslant 45)$ left ventricular ejection fraction

\begin{tabular}{lcccc}
\hline Genotypes/Alleles & $>45 \%$ & $\leqslant 45 \%$ & P-value & OR $(95 \%$ CI $)$ \\
\hline AA & $102(84.3)$ & $80(65.6)$ & - & Reference \\
AC & $17(14)$ & $29(23.8)$ & $\mathbf{0 . 0 2 3}$ & $\mathbf{2 . 1 8}(\mathbf{1 . 1 2}-\mathbf{4 . 2 7})$ \\
CC & $2(1.7)$ & $13(10.7)$ & $\mathbf{0 . 0 0 5}$ & $\mathbf{8 . 6 2}(\mathbf{1 . 8 8}-\mathbf{3 9 . 3 8})$ \\
A & $221(91.3)$ & $189(77.5)$ & - & Reference \\
C & $21(8.7)$ & $55(22.5)$ & $<\mathbf{0 . 0 0 1}$ & $\mathbf{3 . 1 6}(\mathbf{1 . 8 2}-\mathbf{5 . 4 6})$ \\
\hline
\end{tabular}

Significant values are shown in bold.

$0.001 ; \mathrm{OR}=3.72$ and $p \leqslant 0.001 ; \mathrm{OR}=1.78$ respectively in primary cohort) and ( $p=0.001 ; \mathrm{OR}=2.81$ and $p=0.041$; OR $=1.37$ respectively in secondary cohorts) Table 2] On the contrary, no significant difference was observed in the distribution of AT1 A1166C and CYP11B2 T-344C polymorphisms in any of the groups both at genotype and allele levels.

\subsection{Influence of RAAS gene polymorphisms on patients with reduced and preserved ejection fraction}

We segregated CAD patients on the basis of compromised $(\leqslant 45 \%)$ and preserved $(>45 \%)$ left ventricular ejection fraction (LVEF) and compared with their status of $A C E \mathrm{I} / \mathrm{D}, A T 1 \mathrm{~A} 1166 \mathrm{C}$ and $C Y P 11 B 2 \mathrm{~T}-344 \mathrm{C}$ gene polymorphisms. We found that higher percentage of CAD patients carrying heterogeneous AT1 AC and $\mathrm{CC}$ genotypes had compromised ejection fraction ( $\leqslant$ $45 \%$ ) as compared to the patients with preserved ( $>$ $45 \%$ ) ejection fraction. This frequency difference was statistically significant in both the cohorts ( $\mathrm{p}$ value $=$ $0.018, \mathrm{OR}=2.05$ and $\mathrm{p}$ value $=0.013, \mathrm{OR}=3.69$ in AT1 AC and CC genotypes respectively in primary cohort; Table $3 \mathrm{a}$ and $(\mathrm{p}$ value $=0.024, \mathrm{OR}=2.36$ and $\mathrm{p}$ value $=0.020, \mathrm{OR}=5.20 \mathrm{in}$ AT1 $\mathrm{AC}$ and $\mathrm{CC}$ genotypes respectively in secondary cohorts; Table $3 \mathrm{~b}$ ). Also on calculating the overall $\mathrm{z}$ and $\mathrm{p}$ values of both primary and replication cohorts, we found statistically significant values in AT1 CC genotypes $(\mathrm{z}=3.02 ; p \leqslant 0.001$ Table $3 \mathrm{~b})$. No significant difference for $C Y P 11 B 2 \mathrm{~T}-$ 344C gene polymorphism was observed between CAD patients with preserved and compromised LVEF.

We looked for the association of ACE, ATI, CYP11B2 on LVD by changing the cut off values for LVEF to $<40 \%$ and $<50 \%$ also. In case of AT1 the level of significance decreased when we considered the cut off for LV dysfunction to $50 \%$ and at $40 \%$ cut off, level of significance increased but we would have missed some patients with LV dysfunction. Therefore, we selected the cut off to $45 \%$ to include definite patients with LV dysfunction.

\subsection{Distributions for AT1 A1166C gene} Polymorphism in STEMI subjects with preserved $(L V E F>45)$ and compromised $(L V E F \leqslant 45)$ left ventricular ejection fraction

In clinical practice it is well known fact that STEMI patients are more prone to develop LVD. We also observed that $73.1 \%$ of STEMI patients had LVD. Therefore, we looked for distribution of AT1 genotypes in STEMI patients with preserved and compromised ejection fraction. Our results showed that the subjects with CC genotypes and C allele of AT1 polymorphism were more likely to develop LVD as compared to wild type (AA) genotype [Table 4].

Furthermore, we also conducted a separate analysis for stable angina and myocardial infarction (STEMI + NSTEMI) and found that none of the genetic variant of RAAS pathway conferred risk for myocardial infarction.

\section{Discussion}

In the present study we used the candidate gene approach to determine whether the genetic variants of RAAS genes - ACE, $A T 1$ and $C Y P 11 B 2$, being important effectors of the renin-angiotensin-aldosterone system, are involved in LV dysfunction in CAD patients. The main finding of the study indicates close association between AT1 A1166C polymorphism and significantly higher risk of severe left ventricular dysfunction (LVD) in CAD patients.

Remarkably, left ventricular dysfunction (LVD) constitutes the final common pathway for a host of cardiac disorders. Among them, coronary artery narrowing or ischaemic heart disease is the dominant cause of LVD. The LVD produces many changes in the structure and function of the heart through a variety of mechanisms including the renin-angiotensin system, one of the major neuroendocrine axes involved in the development of heart failure [14]. 
Till date, several researchers have investigated the role of AT1 A1166C polymorphism in relation to CAD. Also, there are studies supporting the idea that the ATI $1166 \mathrm{C}$ allele is a predisposing genetic marker for CAD or MI $[4,15,16]$ but there are also reports contrary to these findings $[17,18]$. Our findings show the lack of association of this polymorphism with CAD, as we observed no differences in the prevalence of the ATI $1166 \mathrm{C}$ allele and $\mathrm{CC}$ genotype between CAD patients and healthy controls.

After segregation of CAD patients on the basis of left ventricle ejection fraction (LVEF), the significantly higher ATl CC genotype and 1166C allele frequencies in reduced LVEF subjects point towards an increased risk of LV dysfunction in CAD subjects with LVEF $\leqslant$ $45 \%$.

In humans the effect of angiotensin II (AngII) seems primarily be mediated by AT1 receptors. Increased levels of angiotensin II have been suggested to be involved in the pathophysiology of cardiovascular disease. Angiotensin II, as an acute vasoconstrictor regulates systemic blood pressure and vascular tone. Among several biallelic polymorphisms present in the $A T 1$ receptor gene, A1166C transversion, located at 3' UTR, has been associated with cardiovascular phenotypes such as essential hypertension [19,20], myocardial stiffness [21], and left ventricular hypertrophy [22]. Being present in the non-coding region, the polymorphism can affect gene regulation by interfering with posttranscriptional activities. In a previous study [23] it was reported that $\mathrm{A} 1166 \mathrm{C}$ polymorphism is located within the target sites of miRNA binding. Using reporter silencing assays, it has been shown that miR155 downregulates the expression only of the $1166 \mathrm{~A}$, and not the $1166 \mathrm{C}$ allele of $A T 1$ gene. So in case of $1166 \mathrm{C}$ allele, the expression of AT1 increases, which can lead to arteriolar vasoconstriction and increased blood pressure which ultimately results in reduced cardiac output. There are also reports that density of AT1 receptors increases in myocardial infarction [24]. The increased expression of AT1 receptors in carriers of $1166 \mathrm{C}$ allele, may lead to higher susceptibility for MI, which may later progress to LVD.

Various comparative studies suggest that angiotensin receptor blockers (ARBs) may lower the risk of HF beyond their ability to reduce hypertension. In the field of cardiovascular disease, ACE I/D and the AT1 A1166C gene polymorphisms are probably the most studied genetic variants because the products of these two genes are directly implicated in the pharmacological modulation of the RAAS, and they evidently provoke great interest. In line to this, our data also demonstrates that there is a strong association between ACE I/D gene polymorphism and CAD patients but not with left ventricular dysfunction. Studies on the association of ACE I/D polymorphism and CAD or MI have shown variable results. A recent meta-analysis of 15 studies into the association between the ACE I/D polymorphism and MI in male dominated populations found a mean OR of 1.26 for MI of the DD vs ID+II genotypes (95\% CI $1.15-1.39, p=0.001$ ) [25]. Also few other studies, have suggested a positive association [26,27]. However, a number of other studies have reported negative results [28-30]. Also a previous study demonstrated that the higher level of ACE activity is associated with the development of the moderate left ventricular dysfunction in comparison with ACE activity in patients with preserved left ventricular function. However the severe left ventricular dysfunction $(\mathrm{EF}<40 \%)$ was associated also with low ACE activity [31]. Apart from conferring susceptibility, the ACE gene has been also proposed to play a role in modifying the effect of various treatments in CAD. We also found no association of CYP11B2 T$344 \mathrm{C}$ with either CAD or left ventricular dysfunction. Previous studies on T-344C polymorphism have shown positive [32,33] as well as negative association [34,35] with hypertension and other cardiovascular parameters. There is significant heterogeneity among published reports, with some studies showing association with $\mathrm{T}$ allele [32,36] and others with C allele [37,38]. Since LVD is a polygenic disorder influenced by multiple genes, further association studies and screening of other candidate gene polymorphisms is required to elucidate the precise genetic susceptibility of the disease.

\subsection{Study strengths and limitations}

Being a case-control association study, we have replicated our findings in an independent cohort. Although our sample size is limited, but we have sufficient power $(\beta=0.8)$ to detect a risk of 1.6 or higher. However, it will be worthwhile to replicate the study in different populations before any clinical applications.

In conclusion, our study showed that the ATI 1166C allele is closely associated with left ventricle dysfunction in CAD patients. These findings may have important implications in the understanding of pathobiology of heart failure.

\section{Acknowledgements}

This work was supported by DBT and ICMR, Govt. of India. 


\section{Conflict of Interest}

None declared.

\section{References}

[1] R. McPherson, A. Pertsemlidis, N. Kavaslar et al., A common allele on chromosome 9 associated with coronary heart disease, Science 316 (2007), 1488-1491.

[2] M. Zakrzewski-Jakubiak, S. de Denus, M.P. Dube, F. Belanger, M. White and J. Turgeon, Ten renin-angiotensin systemrelated gene polymorphisms in maximally treated Canadian Caucasian patients with heart failure, Br J Clin Pharmacol 65 (2008), 742-751.

[3] F. Triposkiadis, G. Karayannis, G. Giamouzis, J. Skoularigis, G. Louridas and J. Butler, The sympathetic nervous system in heart failure physiology, pathophysiology, and clinical implications, J Am Coll Cardiol 54 (2009), 1747-1762.

[4] L. Tiret, A. Bonnardeaux, O. Poirier et al., Synergistic effects of angiotensin-converting enzyme and angiotensin-II type 1 receptor gene polymorphisms on risk of myocardial infarction, Lancet 344 (1994), 910-913.

[5] A.P. Pilbrow, B.R. Palmer, C.M. Frampton et al., Angiotensinogen M235T and T174M gene polymorphisms in combination doubles the risk of mortality in heart failure, $\mathrm{Hy}$ pertension 49 (2007), 322-327.

[6] B. Devlin and K. Roeder, Genomic control for association studies, Biometrics 55 (1999), 997-1004.

[7] P.P. Rickham, Human Experimentation. Code of Ethics of the World Medical Association, Declaration of Helsinki, $\mathrm{Br}$ Med J 2 (1964), 177.

[8] N.B. Schiller, P.M. Shah, M. Crawford et al., Recommendations for quantitation of the left ventricle by two-dimensional echocardiography. American Society of Echocardiography Committee on Standards, Subcommittee on Quantitation of Two-Dimensional Echocardiograms, J Am Soc Echocardiogr 2 (1989), 358-367.

[9] R. Johnson, P. McNutt, S. MacMahon and R. Robson, Use of the Friedewald Formula to Estimate LDL-Cholesterol in Patients with Chronic Renal Failure on Dialysis, Clin Chem 43 (1997), 2183-2184.

[10] S.A. Miller, D.D. Dykes and H.F. Polesky, A simple salting out procedure for extracting DNA from human nucleated cells, Nucleic Acids Res 16 (1988), 1215.

[11] G. Joshi, S. Pradhan and B. Mittal, Role of the ACE ID and MTHFR C677T polymorphisms in genetic susceptibility of migraine in a north Indian population, J Neurol Sci 277 (2009), 133-137.

[12] Shu Ye SDXK, A.R Collins and I.N.M Day, An efficient procedure for genotyping single nucleotide polymorphisms, $\mathrm{Nu}$ cleic Acids Research (2001), 29.

[13] A.H. Markku Kupari, L. Lankinen, P. Koskinen, J. Virolainen, H. Nikkila and P.C. White, Associations Between Human Aldosterone Synthase (CYP11B2) Gene Polymorphisms and Left Ventricular Size, Mass, and Function, Circulation 97 (1998), 569-575.

[14] P.W. Armstrong, Left ventricular dysfunction: causes, natural history, and hopes for reversal, Heart 84(Suppl 1) (2000), i15-17: discussion i50.

[15] R. Alvarez, J.R. Reguero, A. Batalla et al., Angiotensinconverting enzyme and angiotensin II receptor 1 polymor- phisms: association with early coronary disease, Cardiovasc Res 40 (1998), 375-379.

[16] I. Canavy, M. Henry, P.E. Morange et al., Genetic polymorphisms and coronary artery disease in the south of France, Thromb Haemost 83 (2000), 212-216.

[17] G.K. Andrikopoulos, D.J. Richter, E.W. Needham et al., The paradoxical association of common polymorphisms of the renin-angiotensin system genes with risk of myocardial infarction, Eur J Cardiovasc Prev Rehabil 11 (2004), 477-483.

[18] M.A. Araujo, B.S. Menezes, C. Lourenco, E.R. Cordeiro, R.R. Gatti and L.R. Goulart, The A1166C polymorphism of the angiotensin II type-1 receptor in acute myocardial infarction, Arq Bras Cardiol 83 (2004), 409-413; 404-408.

[19] Y. Liu, C. Zhuoma, G. Shan et al., A1166C polymorphism of the angiotensin II type 1 receptor gene and essential hypertension in Han, Tibetan and Yi populations, Hypertens Res $\mathbf{2 5}$ (2002), 515-521.

[20] Y. Liu, G.L. Shan, C.Y. Cui et al., [A1166C polymorphism of the angiotensin II type 1 receptor gene and essential hypertension in Han, Tibetan and Yi populations], Zhonghua Yi Xue Yi Chuan Xue Za Zhi 20 (2003), 220-224.

[21] J. Diez, C. Laviades, J. Orbe et al., The A1166C polymorphism of the AT1 receptor gene is associated with collagen type I synthesis and myocardial stiffness in hypertensives, $J$ Hypertens 21 (2003), 2085-2092.

[22] A.P. Osterop, M.J. Kofflard, L.A. Sandkuijl et al., AT1 receptor A/C1166 polymorphism contributes to cardiac hypertrophy in subjects with hypertrophic cardiomyopathy, Hypertension 32 (1998), 825-830.

[23] P. Sethupathy, C. Borel, M. Gagnebin et al., Human microRNA-155 on chromosome 21 differentially interacts with its polymorphic target in the AGTR1 3' untranslated region: a mechanism for functional single-nucleotide polymorphisms related to phenotypes, Am J Hum Genet 81 (2007), 405-413.

[24] M. Maczewski, J. Maczewska and M. Duda, Hypercholesterolaemia exacerbates ventricular remodelling after myocardial infarction in the rat: role of angiotensin II type 1 receptors, $\mathrm{Br}$ J Pharmacol 154 (2008), 1640-1648.

[25] F. Cambien and A. Evans, Angiotensin I converting enzyme gene polymorphism and coronary heart disease, Eur Heart $J$ 16(Suppl K) (1995), 13-22.

[26] B. Keavney, C. McKenzie, S. Parish et al., Large-scale test of hypothesised associations between the angiotensinconverting-enzyme insertion/deletion polymorphism and myocardial infarction in about 5000 cases and 6000 controls. International Studies of Infarct Survival (ISIS) Collaborators, Lancet 355 (2000), 434-442.

[27] K. Lindpaintner, M.A. Pfeffer, R. Kreutz et al., A prospective evaluation of an angiotensin-converting-enzyme gene polymorphism and the risk of ischemic heart disease, $N$ Engl $J$ Med 332 (1995), 706-711.

[28] J.C. Rodriguez-Perez, F. Rodriguez-Esparragon, O. Hernandez-Perera et al., Association of angiotensinogen M235T and A(-6)G gene polymorphisms with coronary heart disease with independence of essential hypertension: the PROCAGENE study. Prospective Cardiac Gene, J Am Coll Cardiol 37 (2001), 1536-1542.

[29] J. Ruiz, H. Blanche, H. Cohen et al., Insertion/deletion polymorphism of the angiotensin-converting enzyme gene is strongly associated with coronary heart disease in non-insulindependent diabetes mellitus, Proc Natl Acad Sci U S A 91 (1994), 3662-3665. 
[30] N.J. Samani, J.R. Thompson, L. O'Toole, K. Channer and K.L. Woods, A meta-analysis of the association of the deletion allele of the angiotensin-converting enzyme gene with myocardial infarction, Circulation 94 (1996), 708-712.

[31] J. Parenica, M.P. Goldbergova, P. Kala et al., ACE gene insertion/deletion polymorphism has a mild influence on the acute development of left ventricular dysfunction in patients with ST elevation myocardial infarction treated with primary PCI, BMC Cardiovasc Disord 10, 60.

[32] E. Brand, N. Chatelain, P. Mulatero et al., Structural analysis and evaluation of the aldosterone synthase gene in hypertension, Hypertension 32 (1998), 198-204.

[33] M. Matsubara, T. Sato, T. Nishimura et al., CYP11B2 polymorphisms and home blood pressure in a population-based cohort in Japanese: the Ohasama study, Hypertens Res 27 (2004), 1-6.

[34] H. Schunkert, S.R. Holmer, U. Broeckel, A. Luchner, M.W. Muscholl et al., Lack of association between a polymorphism of the aldosterone synthase gene and left ventricular structure, Circulation 99 (1999), 2255-2260.

[35] L. Tiret, O. Poirier, V. Nicaud, A. Millaire, J.B. Bouhour et al., Lack of association between polymorphisms of eight candidate genes and idiopathic dilated cardiomyopathy: the CARDIGENE study, J Am Coll Cardiol 35 (2000), 29-35.

[36] E. Davies, C.D. Holloway, M.C. Ingram et al., Aldosterone excretion rate and blood pressure in essential hypertension are related to polymorphic differences in the aldosterone synthase gene CYP11B2, Hypertension 33 (1999), 703-707.

[37] C. Rajput, T. Nnorboo, F. Afrin, M. Sharma, S.T. Pasha et al., CYP11B2 gene polymorphisms and hypertension in highlanders accustomed to high salt intake, J Hypertens 23 (2005), 79-86.

[38] I. Komiya, M. Takara, T. Asawa, M. Shimabukuro, T. Nnishimori et al., Lys(173)Arg and -344T/C variants of CYP11B2 in Japanese patients with low-renin hypertension, Hypertension 35 (2000), 699-703. 


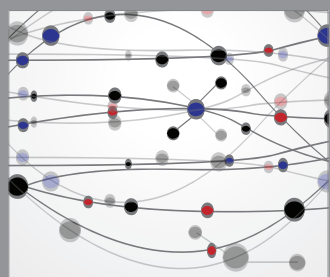

The Scientific World Journal
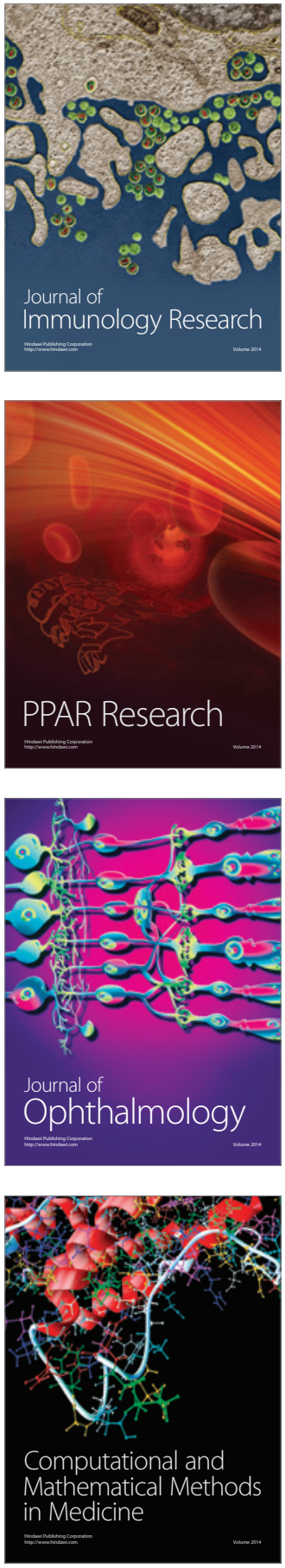

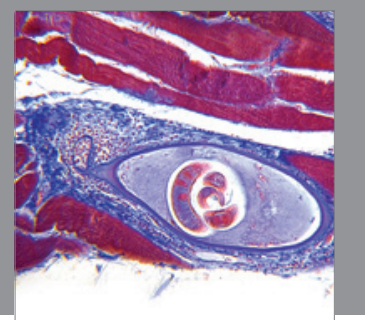

Gastroenterology

Research and Practice
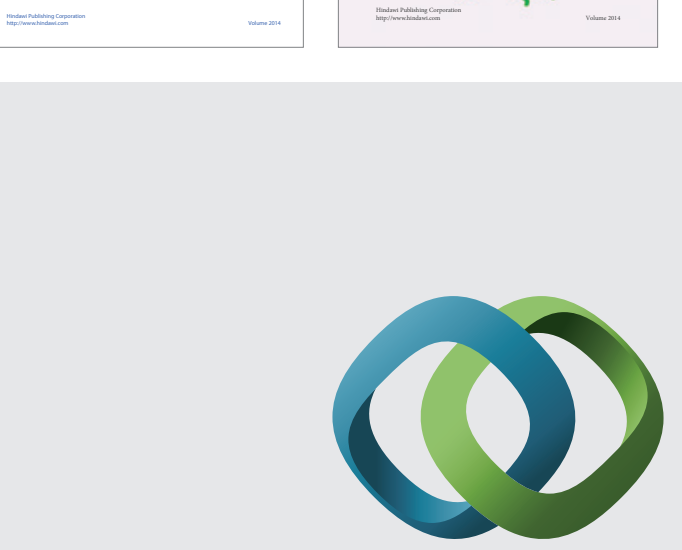

\section{Hindawi}

Submit your manuscripts at

http://www.hindawi.com
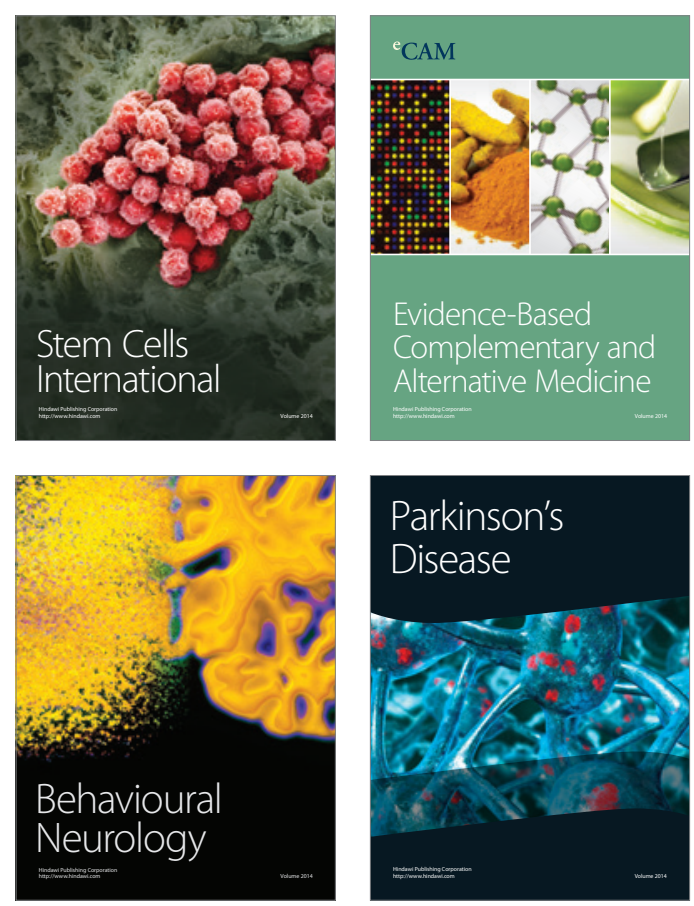

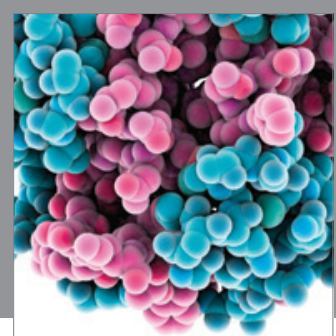

Journal of
Diabetes Research

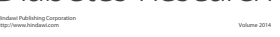

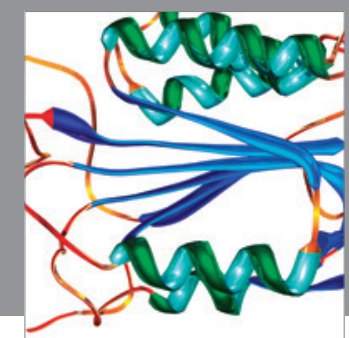

Disease Markers
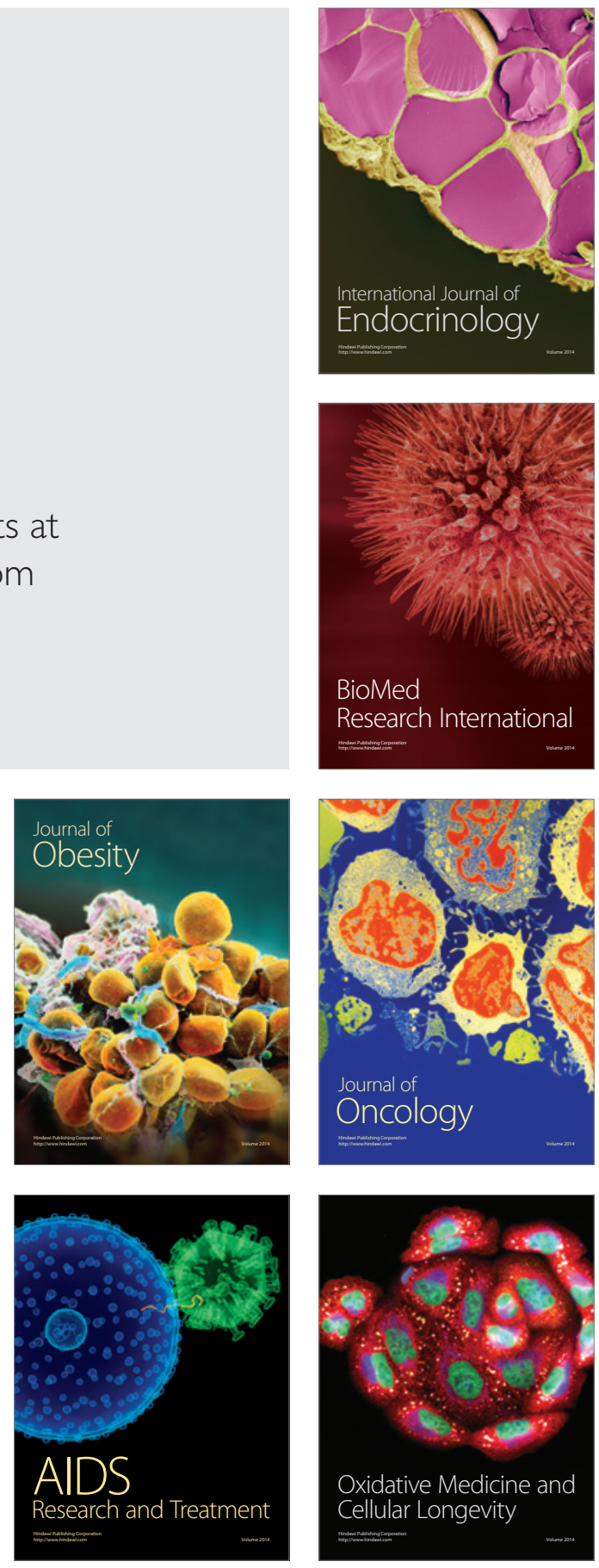